\title{
Participation and performance trends in ultra-triathlons from 1985 to 2009
}

1. B. Knechtle ${ }^{1,2}$,

2. P. Knechtle ${ }^{1}$,

3. R. Lepers ${ }^{3}$

Article first published online: 6 JUL 2010

DOI: $10.1111 / \mathrm{j} .1600-0838.2010 .01160 . \mathrm{x}$

(C) 2010 John Wiley \& Sons A/S

Issue

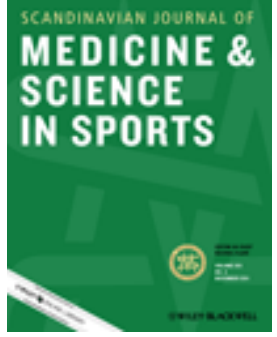

\section{Scandinavian Journal of Medicine \& Science in Sports}

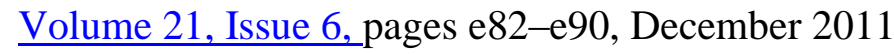

Additional Information(Show All)

$\underline{\text { How to CiteAuthor InformationPublication History }}$

How to Cite

Knechtle, B., Knechtle, P. and Lepers, R. (2011), Participation and performance trends in ultra-triathlons from 1985 to 2009. Scandinavian Journal of Medicine \& Science in Sports, 21: e82-e90. doi: 10.1111/j.1600-0838.2010.01160.x

\section{Author Information}

1. 1

Facharzt FMH für Allgemeinmedizin, Gesundheitszentrum St. Gallen, St. Gallen, Switzerland

2. 2 
Institute of General Practice and Health Services Research, University of Zurich, Zurich, Switzerland

3. 3

INSERM U887, Faculty of Sport Sciences, University of Burgundy, Dijon, France

*Corresponding author: Beat Knechtle, Facharzt FMH für Allgemeinmedizin,

Gesundheitszentrum St. Gallen, Vadianstrasse 26, 9001 St. Gallen, Switzerland. Tel: +4171 22682 82, Fax: +41 7122682 72, E-mail: beat.knechtle@ hispeed.ch

\section{Publication History}

1. Issue published online: 29 NOV 2011

2. Article first published online: 6 JUL 2010

3. Accepted for publication 10 May 2010

\section{SEARCH}

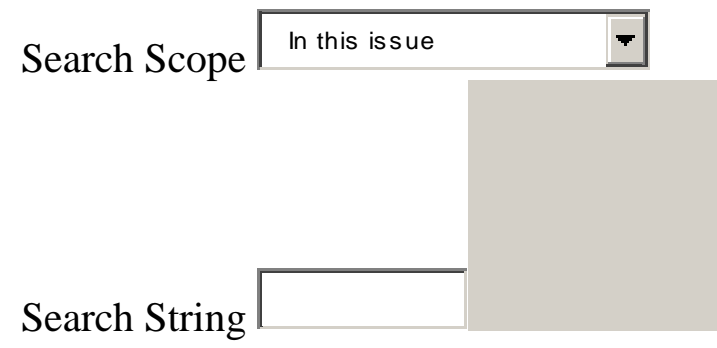

- Advanced $>$

- $\quad$ Saved Searches $>$

\section{SEARCH BY CITATION}

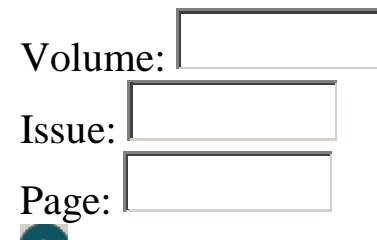

\section{ARTICLE TOOLS}

- Get PDF (344K)

- $\quad$ Save to My Profile

- E-mail Link to this Article

- Export Citation for this Article

- Get Citation Alerts

- Request Permissions 
More Sharing ServicesShare|Share on citeulikeShare on connoteaShare on deliciousShare on www.mendeley.comShare on twitter

- $\underline{\text { Abstract }}$

- Article

- References

- Cited By

\section{$\underline{\text { Get PDF (344K) }}$}

\section{Keywords:}

- gender;

- sex difference;

- swimming;

- cycling;

- running;

- endurance

Jump to...

\section{Abstract}

1. Top of page

2. Abstract

3. Material and methods

4. Results

5. Discussion

6. Perspectives

7. Acknowledgements

8. References

We examined the changes in participation and performance trends in ultra-triathlons, from the Double Iron (7.6 km swimming, $360 \mathrm{~km}$ cycling, $84.4 \mathrm{~km}$ running) to the Deca Iron $(38 \mathrm{~km}$ swimming, $1800 \mathrm{~km}$ cycling, $422 \mathrm{~km}$ running), between 1985 (first year of a Double Iron) and 2009 ( 25 years). The mean finish rate for all distances and races was $75.8 \%$. Women accounted for $\sim 8-10 \%$ of the ultra-triathlons starters. For Double and Triple Iron, the number of finishers per year increased, from 17 to 98 and from 7 to 41, respectively. In the Deca Iron, the finishers per race have remained $<20$ since the first event was held, up to 2009.

Concerning World best performances, the men were $\sim 19 \%$ faster than the women in both the Double and Triple Iron, and $\sim 30 \%$ faster in a Deca Iron. With the increasing length of ultratriathlons, the best women became relatively slower compared with the best men. Further 
investigations are required to understand why this gender difference in total performance time increased with the distance in ultra-triathlons.

The discipline of triathlon involves successively swimming, cycling and running. The traditional distances vary from the short (or Olympic) distance, i.e. $1.5 \mathrm{~km}$ swimming, $40 \mathrm{~km}$ cycling and $10 \mathrm{~km}$ running, to the Ironman distance, i.e. of $3.8 \mathrm{~km}$ swimming, $180 \mathrm{~km}$ cycling and $42.2 \mathrm{~km}$ running (Lepers, 2008). Triathlons of greater distances than the Ironman are usually called "ultra-triathlons," such as the Double Ironman triathlon (2IMT) distance of $7.6 \mathrm{~km}$ swimming, $360 \mathrm{~km}$ cycling and $84.4 \mathrm{~km}$ running (Lehmann et al., 1995; Gastmann et al., 1998), the Triple Ironman triathlon (3IMT) distance of $11.4 \mathrm{~km}$ swimming, $540 \mathrm{~km}$ cycling and $126.6 \mathrm{~km}$ running (Knechtle et al., 2007a; Knechtle \& Kohler, 2009), and the Deca Ironman triathlon (10IMT) distance of $38 \mathrm{~km}$ swimming, $1800 \mathrm{~km}$ cycling and $420 \mathrm{~km}$ running (Knechtle et al., 2007b).

The Ironman distance is the most popular long-distance triathlon. Since the first event was held in 1978, participation has increased, and nowadays thousands of triathletes compete in about 25 Ironman races all over the world in order to qualify for the Ironman Hawaii, where each year more than 1700 triathletes participate in the Ironman triathlon World Championship (Lepers, 2008; Lepers et al., 2010). The first ultra-triathlons, of distances greater than the Ironman distance, appeared since 1985. Historically, the first 2IMT was held in 1985 in Huntsville (USA) where 23 athletes started. Then, in 1988, the first 3IMT was organized in Le Fontanil (France) with 11 starters. The first Quadruple Ironman triathlon (4IMT) was held in 1989 in Den Haag (the Netherland) where two athletes started, and the first Quintuple Ironman triathlon (5IMT) was held in 1991, again in Den Haag (the Netherland), with 10 starters. In 1992 the ultra-triathlon scene moved to Monterrey (Mexico) where 20 athletes started in the first 10IMT.

In the last two decades the ultra-endurance events, especially ultra-running and Ironman triathlon, have become more and more popular throughout the world (Lepers, 2008; Hoffman \& Wegelin, 2009; Hoffman, 2010; Lepers et al., 2010). Participation and performance trends have previously been investigated for ultra-marathons (Hoffman \& Wegelin, 2009; Hoffman, $\underline{2010}$ ) and triathlons (Lepers, 2008), but not for ultra-triathlons. Hoffmann and colleagues analyzed participation and performance trends in 100-mile ultra-marathons (Hoffman \& Wegelin, 2009; Hoffman, 2010). In the "Western States 100-Mile Endurance Run," they found an increase in starters from 1974 to 1986, and thereafter a stable entry. In 1986, the first women competed in that race. The participation among women has increased from $10-12 \%$ in the late 1980s to 20-22\% where it has remained since 2001 (Hoffman \& Wegelin, 2009). In the Hawaii Ironman triathlon, nowadays, $27 \%$ of the participants are women. The number of total starters at the Western States Endurance Run and at the Hawaii Ironman triathlon have 
been stable since 1990s because of a limitation in the number of entries than can be accepted rather than a lack of interest in participating in these events.

It has been suggested that gender differences in running would disappear over longer distances. Sparling et al. (1998) analyzed the World best times from the 1980s to 1996 for the $1500 \mathrm{~m}$ and marathon distances. In 1980, the marathon was a fairly new event for women. After an initial improvement, the best times for women have leveled off and remain $\sim 11 \%$ behind the men. Coast et al. (2004)investigated the distances between $100 \mathrm{~m}$ and $200 \mathrm{~km}$. Also over longer distances speeds were different, with men being $~ 12 \%$ faster than women. Indeed, longer distances were associated with greater differences. Regarding the ultramarathon distances, the finishing times of women improved relative to men throughout the 1980 s, but then stablized over the past two decades with the fastest women running 20\% slower than the fastest men. Interestingly, the average times of the fastest runners have not changed over the past two decades for either gender ( $\underline{\text { Hoffman, 2010)}}$.

The aim of this study was to investigate the participation and performance trends of both men and women over the ultra-triathlon distances, ranging from 2IMT to 10IMT, from the beginning in 1985 until 2009. In the analysis of Ironman performances at the Ironman Hawaii from 1981 to 2007, performance times decreased in the beginning, but reached a plateau within 10 years (Lepers, 2008). Gender differences for the split times in swimming, cycling and running, and the total race time, were $\sim 10 \%$ (Lepers, 2008). We hypothesized that for longer distances up to the 10IMT, there would be an improvement in performance over the 25 years, and male athletes would remain faster compared with women athletes.

Jump to...

\section{Material and methods}

1. Top of page

2. Abstract

3. Material and methods

4. Results

5. Discussion

6. $\underline{\text { Perspectives }}$

7. Acknowledgements

8. References

All the race results from 2IMT to 10IMT, showing total times and the split times for swimming, cycling and running, were collected from 1985 to 2009 by two of the authors. All the race results were provided by the organizers of the different races. The total number of 
starts and finishes were considered even if some people likely completed multiple events. Linear regressions were used for estimating the changes of selected variables (total, swimming, cycling and running performances for both men and women winner of each race) across years. One-way ANOVA was used to compare gender difference in swimming, cycling, running and total time across ultra-triathlons. Data analysis was approved by the Ethical Committee of the Canton, St. Gallen, Switzerland.

Jump to...

\section{Results}

1. Top of page

2. Abstract

3. Material and methods

4. $\underline{\text { Results }}$

5. Discussion

6. Perspectives

7. Acknowledgements

8. References

\section{Participation}

The total number of starts and finishes for both men and women in the different ultratriathlons, since the first year each event was held, are given in Table 1. The 4IMT and 5IMT appear to have been considered as "experimental" on the way to the longest ultra-triathlon distance, i.e. the 10IMT, and so the number of race starts and finishes in these events have been very small compared with the other ultra-triathlon distances. Therefore, we focused only on the 2IMT, 3IMT and 10IMT in the following results.

Table 1. Total number of races, year of first race, total number of starts and finishes, and mean finish rate $( \pm$ SD) for 2IMT, 3IMT, 4IMT, 5IMT and 10IMT from the year of the first race to 2009, inclusive
Numbe Yea
Overall
Men
Women 


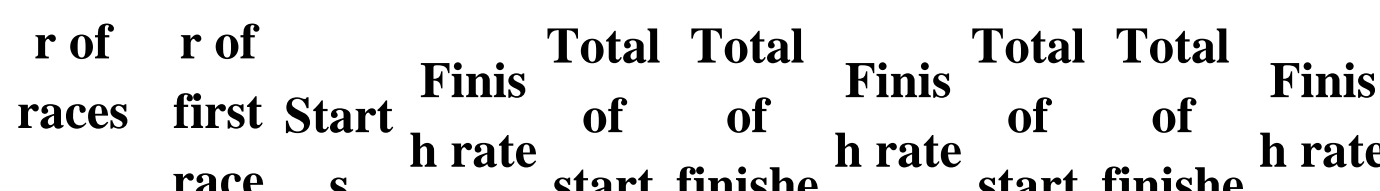 race $\mathbf{S}$ (\%) start finishe $(\%)$ \\ $\mathbf{S} \quad \mathbf{S}$ \\ $\mathbf{S}$ \\ $\mathbf{S}$ \\ (\%)}

1. 2IMT, Double Ironman triathlon; 3IMT, Triple Ironman triathlon; 4IMT, Quadruple Iron triathlon; 5IMT, Quintuple Iron triathlon; 10IMT, Deca Ironman triathlon.

2IMT

(7.6-

360

$85 \quad 19851686^{79.5 \pm} 6.7 \quad 1549$

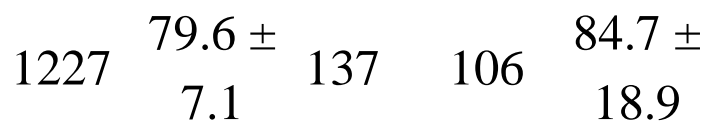

$84 \mathrm{~km})$

3IMT

$(11.4-$

540

$126 \mathrm{~km}$

$\begin{array}{llllllllll}48 & 1988 & 1101 & \begin{array}{c}72.2 \pm \\ 10.5\end{array} & 1009 & 724 & \begin{array}{c}71.7 \pm \\ 10.8\end{array} & 92 & 72 & 77.0 \pm \\ & & & & & \end{array}$

)

4IMT

(15.2-

$720-$

$168 \mathrm{~km}$

$\begin{array}{lllc}5 & 1989 & 64 & \begin{array}{c}68.7 \pm \\ 18.5\end{array} 62\end{array}$

$\begin{array}{ccc}41 & 67.9 \pm & 2\end{array}$

$\begin{array}{cc}2 & 100.0 \\ & \pm 0\end{array}$

)

5IMT

(19-

900-

$210 \mathrm{~km}$

$\begin{array}{lllcl}4 & 1991 & 59 & 80.1 \pm \\ 21.1\end{array} 49 \quad 36$

$83.1 \pm 10 \quad 9$
19.5

$95.0 \pm$

8.7

)

10IMT

(38-

1800

$420 \mathrm{~km}$

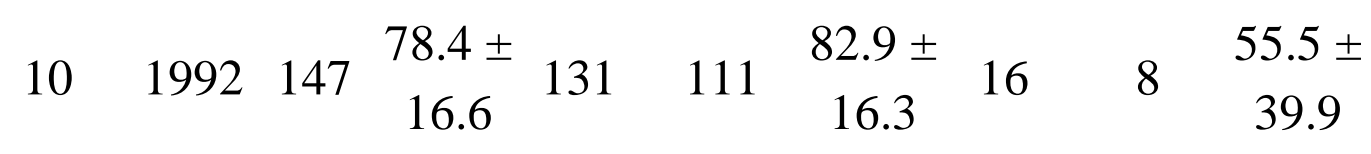


The number of starts in the 2IMT has progressively increased from 1985 to 2009 (number of starts in 1985: 23 men, 0 women; number of starts in 2009: 109 men, 12 women). Women accounted for $\sim 10 \%$ of the 2IMT starts since 2000 . The number of starts in the 3IMT has also increased since 1988: men starts ranging from 11 (in 1998) to 69 (in 2000) and women starts ranging from 0 (in 1998) to 11 (in 1997). Women accounted for $~ 8 \%$ of the 3IMT starts since 1988. For 10IMT, the number of men starts ranged from 4 in 1998 to 24 in 2004 and the number of women starts ranged from 0 in 1995 to 3 in 2004. Women accounted for $\sim 10 \%$ of 10IMT starts since 1992.

Figure 1 shows the changes in women and men finishers in the 2IMT, 3IMT and 10IMT distances since the first year of each race. For the 2IMT and 3IMT the total number of finishers has increased since the first year. In contrast, in the 10IMT, the total number of finishers decreased after the first year, until 1998, and then increased thereafter, until stabilizing from 2004.

Figure 1. Total number of finishers, and finishers of each sex, in the 2IMT from 1985 to 2009 (a), in the 3IMT from 1988 to 2009 (b) and in the 10IMT from 1992 to 2008 (c). The 10IMT was only held on even years. 2IMT, Double Ironman triathlon; 3IMT, Triple Ironman triathlon; 10IMT, Deca Ironman triathlon.

$\underline{\text { Download figure to PowerPoint }}$

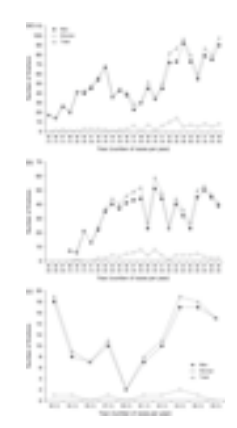

\section{Performance}

Table 2presents the actual World best times in the 2IMT, 3IMT and 10IMT, for both men and women, together with the corresponding swim, cycle and run split times. When the World best total performance times were compared, gender differences were similar for the 2IMT and 3IMT $(\sim 19 \%)$, while it was greatest for 10IMT $(\sim 30 \%)$.

Table 2. World best total event performance times for men and women with corresponding split times for swimming, cycling and running for 2IMT, 3IMT and 10IMT 


\section{Country Total race Swim time Cycle time Run time (year) time $(\min ) \quad(\min ) \quad(\min ) \quad(\min )$}

1. Gender difference is expressed as a percentage of the men's time.

2. 2IMT, Double Ironman triathlon; 3IMT, Triple Ironman triathlon; 10IMT, Deca Ironman triathlon.

2IMT

$\begin{array}{llllll}\text { Men } & \begin{array}{l}\text { Belgium } \\ (1993)\end{array} & 1194 & 124 & 628 & 441 \\ \text { Women } & \begin{array}{l}\text { Belgium } \\ (1992)\end{array} & 1425 & 165 & 761 & 499\end{array}$

Difference

$(\%)$

$19.3 \quad 33.1 \quad 21.2 \quad 13.2$

3IMT

Men

Germany

(2003)

$1907 \quad 229 \quad 987 \quad 690$

Women

Germany

(1996)

2274

236

1215

823

Difference

(\%)

19.2

3.1

23.1

19.3

10IMT

$\begin{array}{llllll}\text { Men } & \begin{array}{l}\text { Mexico } \\ (1997)\end{array} & 11528 & 671 & 6026 & 4829 \\ \text { Women } & \begin{array}{l}\text { Mexico } \\ (1992)\end{array} & 14954 & 1383 & 7194 & 6376\end{array}$

Difference

(\%)

29.7

$106.1 \quad 19.4$

32.0

Figure 2 shows the historical performance trends of the women and men winners in the 2IMT from 1985 to 2009. Regression lines are presented for the total time, together with the three 
disciplines, for both sexes. The gradients of the regression lines demonstrate that swimming, cycling, running and total performance has not significantly changed over the last 25 years for both men and women.

Figure 2. Total, swimming, cycling and running performance times in the 2IMT, for both men and women winners, from 1985 to 2009. All winners were considered when several races took place the same year. Regression lines are presented as a bold line for men and a dotted line for women. 2IMT, Double Ironman triathlon.

\section{Download figure to PowerPoint}

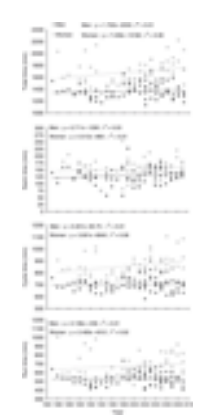

The historical performance trends for both women and men winners in the 3IMT, from 1988 to 2009, are presented in Fig. 3. For men, the gradients of the regression lines demonstrate that cycling, running and total performances have not changed since 1988. In contrast, swimming performance times have tended to increase over this period. Surprisingly, for women, the total and running performance times have increased since 1988, while the swimming and cycling performances have remained stable over these last two decades.

Figure 3. Total, swimming, cycling and running performance times in the 3IMT, for both men and women winners, from 1988 to 2009. Regression lines are presented as a bold line for men and a dotted line for women. 3IMT, Triple Ironman triathlon.

\section{Download figure to PowerPoint}

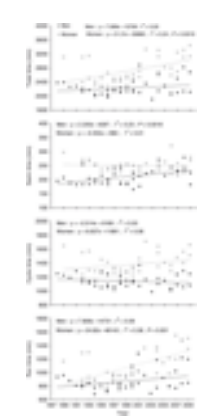


Figure 4 shows the changes in performance times for both the women and men winners in the ten 10IMT held since 1992. The total swimming, cycling and running performances have not significantly changed since the first event for either men or women.

Figure 4. Total, swimming, cycling and running performance times in the 10IMT, for both men and women winners, from 1992 to 2008. No race took place in 2009. Regression lines are presented as a bold line for men and a dotted line for women. 10IMT, Deca Ironman triathlon.

\section{Download figure to PowerPoint}

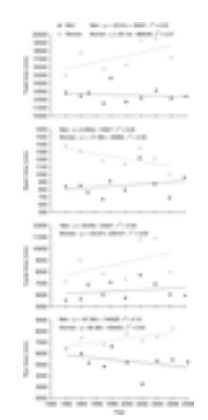

The average differences in the total, and in the swimming, cycling and running, times between the women and men winners at 2IMT, 3IMT and 10IMT are presented in Table 3. Gender differences for the total distances, together with the three disciplines, are in the same range for both the 2IMT and 3IMT, but are significantly greater for the 10IMT when compared with the 2IMT and 3IMT, except for running.

Table 3. Differences in total swimming, cycling and running times between the female and male winners for all 2IMT, 3IMT and 10IMT races with at least one women finisher since the first event (mean \pm CI 95\%)

$$
\text { Total (\%) Swim (\%) } \quad \text { Cycle (\%) } \quad \text { Run (\%) }
$$

1. Gender differences are expressed as a percentage of men's time. ${ }^{*} P<0.05,{ }^{* *} P<0.01$ : significantly different from 2IMT. $\$ P<0.05, \$ \$ P<0.01$ : significantly different from 3IMT. 2IMT, Double Ironman triathlon; 3IMT, Triple Ironman triathlon; 10IMT, Deca Ironman triathlon.

$\begin{array}{lllll}\text { 2IMT }(n=56) & 25.2 \pm 3.8 & 21.8 \pm 7.8 & 23.3 \pm 3.3 & 30.3 \pm 6.6 \\ \text { 3IMT }(n=36) & 21.4 \pm 4.9 & 19.3 \pm 6.1 & 20.3 \pm 4.2 & 24.6 \pm 8.1\end{array}$


Table 3. Differences in total swimming, cycling and running times between the female and male winners for all 2IMT, 3IMT and 10IMT races with at least one women finisher since the first event (mean $\pm \mathrm{CI}$ 95\%)

\begin{tabular}{|c|c|c|c|c|}
\hline & Total $(\%)$ & Swim (\%) & Cycle (\%) & Run (\%) \\
\hline & $40.3 \pm 11.8 \stackrel{* \$ \$ \$}{*}$ & $47.4 \pm 19.3^{*}, \$$ & $39.5 \pm 12.5 \stackrel{* * \$ \$ \$}{ }$ & $45.9 \pm 33.9$ \\
\hline
\end{tabular}

The trends in the top five men's times' spreads are shown in Fig. 5 for all the 2IMT, 3IMT and 10IMT held since 1985. The gradients of the regression lines demonstrate that the top five times' spreads have not changed since the first event over any distance.

Figure 5. Top five times' spreads for men across the times for the 2IMT, 3IMT and 10IMT with linear regressions shown by the solid lines. The gradients of the three distances were not different from zero. 2IMT, Double Ironman triathlon; 3IMT, Triple Ironman triathlon; 10IMT, Deca Ironman triathlon.

$\underline{\text { Download figure to PowerPoint }}$

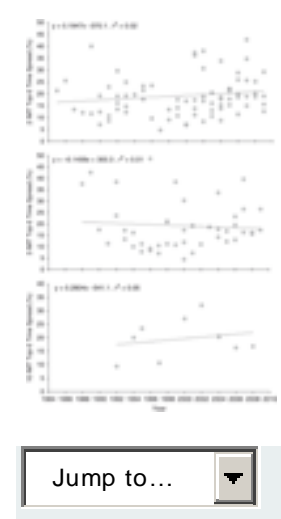

\section{Discussion}

1. Top of page

2. Abstract

3. Material and methods

4. Results

5. Discussion

6. Perspectives

7. Acknowledgements

8. References 
The aim of this study was to investigate the participation and performance trends at ultratriathlon events from 1985 to 2009 . We hypothesized that an improvement in performance over the 25 years would occur, and that male athletes would be faster compared with female athletes. Apart from the study of Lepers (2008) at the Ironman Hawaii, and the studies from Hoffman and colleagues (Hoffman \& Wegelin, 2009; Hoffman, 2010) in the 100-miles ultramarathons in North America, this is the first study to describe the development of ultraendurance events over 25 years, where the athletes pushed themselves to the limits of ultraendurance performance. Interestingly, the length of the races for ultra-triathletes increased within a short period of time.

\section{Participation and finisher rates}

In 1985 the first 2IMT was held. Within 7 years the athletes had moved up to the 3IMT, the 4IMT and the 5IMT, and ultimately to the 10IMT. Owing to the low number of races over the 4IMT and 5IMT distances, we focused our analysis on the 2IMT, 3IMT and 10IMT. While the organization of a 2IMT and 3IMT is not an insoluble logistic problem, distance of more than the 3IMT require a closed circuit to traffic regarding the rules of the International UltraTriathlon Association. The only organizer who is able to offer for weeks a closed circuit is the organizer in Monterrey, Mexico, where nearly all races of 5IMT and longer were held (Knechtle et al., 2007b).

With the increasing length of the races, the number of participants dramatically decreased. The rate of male finishers in all distances was $\sim 80 \%$, whereas it varied - due to the low number of participants - in females, between $55 \%$ for the 10IMT and $100 \%$ for the 4IMT.

In the "Western States 100-Mile Endurance Run" the participation of women increased between 1986 and 2007 to $20 \%$ of all starters (Hoffman \& Wegelin, 2009). The overall yearly finish rates have ranged from $48 \%$ to $80 \%$ since 1978 , and the overall finish rate for men $(64 \%)$ was comparable to that of women $(62 \%)$. When all the 100-mile ultra-marathons in North America were analyzed, the average finisher rate was $\sim 60 \%$ (Hoffman, 2010). For the ultra-triathletes, however, the mean finish rate was higher for both men $(\sim 78 \%)$ and women $(\sim 72 \%)$ over all the ultra-triathlon distances, compared with the 100-mile ultramarathoners.

In the "Western States 100-Mile Endurance Run," the average yearly number of starters was around 300 men and 50 women runners (Hoffman \& Wegelin, 2009). With a total distance of $161 \mathrm{~km}$ and an average finish time between 25 and $30 \mathrm{~h}$, the "Western States 100-Mile Endurance Run" is comparable to the shortest distance in ultra-triathlon, i.e. the 2IMT. Most 
of the ultra-triathletes participated in the 2IMT and in 3IMT. These two distances can be completed without sleep and at a rather high speed (Knechtle et al., 2007a, 2008a).

In ultra-triathlons, female participation was lower compared with the 100-mile ultramarathoners (Hoffman \& Wegelin, 2009). The number of women starters in the 2IMT has slightly increased and accounted for $\sim 10 \%$ in the last 10 years; for the 3IMT and 10IMT, women starters were, from the beginning of the events, at $8 \%$ and $10 \%$, respectively. In the Ironman Hawaii, however, the number of women increased progressively from 20 in 1981 (6\% of the participants) to more than 450 in 2007 (27\% of the participants). Also in the "Western States 100-Mile Endurance Run" there was an increasing participation among women, to 20\% of all starters between 1986 and 2007 (Hoffman \& Wegelin, 2009).

\section{Performance trends}

Regarding gender differences in performance, Lippi et al. (2008) analyzed the World records, including runs up to the marathon distance, from 1900 to 2007 where, overall, the relative improvement in athletic performance was higher in women than in men. Considering the World best performances in marathon running, men were $\sim 11 \%$ faster then women from 1980 to 1996 (Sparling et al., 1998).

In ultra-endurance performances, there is still a debate about whether women could be faster than men. Bam et al. (1997) evaluated both men and women ultra-marathon performances and found that men ran faster over $5-42.2 \mathrm{~km}$, but not in a $90 \mathrm{~km}$ ultra-marathon. It is, indeed, possible that a women ultra-runner can win a multi-stage ultra-marathon ahead of all the male competitors (Knechtle et al., 2008b). When performances over $100 \mathrm{~m}$ to $200 \mathrm{~km}$ between men and women were compared, men were, however, averaged $12.4 \%$ faster compared with women (Coast et al., 2004). Also in a 100-mile ultra-marathon, the fastest women were $20 \%$ slower compared with the fastest men (Hoffman, 2010).

In the 2IMT and 3IMT, the World best performance times for men were $19.3 \%$ and $19.2 \%$ faster compared with women. For all winners, men were $25.6 \%$ faster in the 2 IMT and $21.4 \%$ faster in the 3IMT compared with women. For the 10IMT, World best performance was $29.7 \%$ faster for men. For all winners, men were $40.3 \%$ faster than women. The winner gender differences in performance times for both the 2IMT and 3IMT $(\sim 19 \%)$ are slightly greater comparable to those found for an Ironman ( 13\%) (Lepers, 2008). In all distances greater than the Ironman distance, women became progressively slower than men. This finding is in accordance with the findings for runners when distances between $100 \mathrm{~m}$ and $200 \mathrm{~km}$ were investigated (Coast et al., 2004). It is possible that non-physiological factors may have contributed to these observations. For example, the widening of gender difference 
with increase distance could be due at least in part to the fewer number of women triathletes in 10IMT.

\section{Performance in swimming, cycling and running}

Considering total race time and the split times, male winners were $\sim 20 \%$ faster for total race time, including split times, in the 2IMT and 3IMT. In the run split for the 2IMT, male winners were $\sim 30 \%$ faster than female winners. In the 10IMT, male winners were $\sim 40 \%$ faster than females. In both the swim and run splits, male winners were $\sim 45 \%$ faster than female winners. Regarding performance, neither split times nor the total race time became faster for men in the 2IMT, 3IMT and 10IMT during these 25 years. For the split times in the 3IMT, from 1988 to 2009, running performance increased for female winners and swimming performance increased for male winners. In the 2IMT and 10IMT, no changes for split times and total performances were found. For ultra-runners, Hoffman (2010) showed that the average times of the fastest runners had not changed over the last 20 years in the $161 \mathrm{~km}$ ultra-marathon in the United States. Also for the top five finishers among all men, or within different age groups, no changes could be detected (Hoffman \& Wegelin, 2009).

The sport of triathlon involves three separate disciplines. In the Ironman Hawaii, between 1998 and 2007, the gender difference remained stable and practically identical for swimming $(+0.1 \%$ per decade), increased a little for cycling $(+0.8 \%$ per decade $)$ and decreased somewhat more for running (-2.8\%) (Lepers, 2008). In the 2IMT and 3IMT, the gender difference in time was the smallest in swimming and the greatest in running for both men and women winners. In the 10IMT, however, gender difference was the smallest in cycling.

In the Ironman Hawaii, time performances for the three disciplines, and for both sexes, decreased during the 1980s and tended to stabilize over the last two decades (Lepers, 2008). From 1988 to 2007, the change in swimming, cycling, running and total performance for both men and women was $<1.4 \%$ per decade, except for the women's run time, which decreased by $3.8 \%$ per decade. So while running performance for women improved in the Ironman Hawaii, it declined in the 3IMT.

A further important finding was that with the exception of the World best time in the 3IMT for men, all the World best times were achieved in the early or mid 1990s. In 2000 no improvement in the World best times occurred for any distance. This finding is in line with global findings in the development of World records. When all the World records for Olympic disciplines were evaluated, a major global fading of World record progression was found (Berthelot et al., 2008). 


\section{How could these gender differences be explained?}

It is well known that women are different regarding anthropometry, with women having more fat mass and less skeletal muscle mass compared with men (Sanborn \& Jankowski, 1994). During ultra-endurance races such as the 3IMT, a loss of both muscle mass and fat mass has been described (Knechtle et al., 2008a). The decrease in skeletal muscle mass occurs in both women (Knechtle et al., 2008a) and men (Knechtle et al., 2008b) ultra-endurance athletes. When we consider that performance times increased for women with the increasing length in the ultra-distance races, we must assume that the smaller muscle mass at the start, and the decrease during the ultra-race, must be the limiting factor. Already in 1998, when the World best times from $1500 \mathrm{~m}$ to the marathon were compared, Sparling et al. (1998) concluded that the differences in performance will remain due to the biological differences between men and women.

Jump to...

\section{Perspectives}

1. Top of page

2. Abstract

3. Material and methods

4. $\underline{\text { Results }}$

5. Discussion

6. Perspectives

7. Acknowledgements

8. References

Within 7 years, from 1985 to 1992, ultra-triathletes had moved progressively from the 2IMT to the 10IMT. While the 2IMT and 3IMT are of increasing popularity, the 4IMT and 5IMT seemed to be experimental toward a move to the 10IMT. Since performance in the 2IMT, 3IMT and 10IMT showed no improvement over 25 years, we assume that only ultraendurance athletes of a very high level started ultra-triathlons from the initiation of the sport. Presumably, ultra-triathletes enter the scene in distances such as the 2IMT and 3IMT and then move later to the 10IMT. However, it is not known whether the best ultra-triathletes competing in the 2IMT and 3IMT will also be the best in the 10IMT. The relationship between the 2IMT and 3IMT performances to the 10IMT performances for the same athletes need to be further investigated. The mean finish rate for all distances was $75 \%$, which is high compared with a $161 \mathrm{~km}$ ultra-marathon. With the increasing length of the races, women became relatively slower compared with men. So women seem not to be able to compete 
faster than men in ultra-endurance performances. With the increasing length of the races, the smaller skeletal muscle mass in women may be a limiting factor. Further investigations are required in order to understand why gender differences in total performance times increase with the distance in ultra-triathlons.

Jump to...

\section{Acknowledgements}

1. Top of page

2. $\underline{\text { Abstract }}$

3. Material and methods

4. $\underline{\text { Results }}$

5. Discussion

6. Perspectives

7. Acknowledgements

8. References

We thank May Miller, Stockton-on-Tees, Cleveland, UK, for her help in English translation. 